\title{
Spontaneous emission spectra of organic molecules in synthetic opal photonic crystals
}

\author{
Guziy O.O. ${ }^{1}$, Abu Sal B. ${ }^{2}$, Moiseyenko V.N. ${ }^{1}$, Dergachov M.P. ${ }^{1}$ and \\ Shvachich V.G. ${ }^{1}$ \\ ${ }^{1}$ Dnipropetrovsk National University, 72 Gagarin Ave., \\ 49050 Dnipropetrovsk, Ukraine,e-mail: optic@ff.dsu.dp.ua \\ ${ }^{2}$ Department of Science, Taibah University, Kingdom of Saudi Arabia
}

Received: 15.05 .2008

\begin{abstract}
The emission of rhodamine $6 \mathrm{G}$ and 2,5-bis(2-benzoxazolyl)hydroquinone molecules embedded in synthetic opal photonic crystals are studied in the spectral range of $520-620 \mathrm{~nm}$. It is found that the intensity of spontaneous emission is suppressed in the spectral region corresponding to the photonic stop band and is amplified on its "blue" side.
\end{abstract}

PACS: 78.67-n, 78.55.Mb

UDC: 535.361

Keywords: photonic crystals, photonic forbidden gap, stop band, fluorescence spectra, photon density of states

\section{Introduction}

A great interest to optical phenomena occurring in photonic band-gap structures or photonic crystals is caused by the existence of forbidden gaps in their photonic band structure [1-3]. These gaps represent frequency regions where electromagnetic waves are forbidden, irrespective of the spatial propagation direction. Inside the bands, the photon density of states (DOS) is equal to zero and so the emission of light sources embedded in these crystals should be suppressed in these spectral regions. Since the time the effect is predicted, many experiments have been devoted to studies of spontaneous emission of molecules embedded in different photonic crystals [4-8]. As a prototype of threedimensional photonic crystals, synthetic opal photonic crystals (SOPC) have been used. They represent artificial three-dimensionally periodic, globular dielectric structures with the period close to the light wavelength. The structures demonstrate both a pronounced stop band (i.e., a forbidden gap actual for one direction) for the case of light propagation along $\langle 111\rangle$ direction and a change in the photon DOS near the stop band. Advances in fabrication of SOPC and a wide range of possible modifications of its optical properties by infiltrating the SOPC with various substances stimulate extensive studies of this material [9].

The purpose of the present work is to study the fluorescence spectra of rhodamine 6G (R6G) and 2,5-bis(2-benzoxazolyl)hydroquinone (BBHQ) molecules embedded in the SOPC. 


\section{Samples and Experimental Setup}

The samples under study were synthetic opal crystals made of $\mathrm{SiO}_{2}$ globules. The existence of periodic structure in such the opals has to be proved by iridescence characteristic of the natural opal. Our samples answered this demand when illuminated by a visible light (see Fig. 1). The dimensions of the volume samples were approximately $1.0 \times 1.0 \times 0.5 \mathrm{~cm}^{3}$ (Fig. 1c). The SOPC samples were doped with R6G and BBHQ molecules by soaking the samples in a dilute ethanol solution $\left(10^{-4} \mathrm{M}\right)$ for the case of R6G or in a dilute 3-metylpentane solution $\left(10^{-3} \mathrm{M}\right)$ for the case of BBHQ. The concentration of the solution for R6G was kept low in order to prevent re-absorption of light and non-radiative transfer. Some of the samples with the embedded R6G molecules were additionally infiltrated with colloidal silver. Besides the volume SOPC samples, film synthetic opals were also infiltrated with the BBHQ molecules.

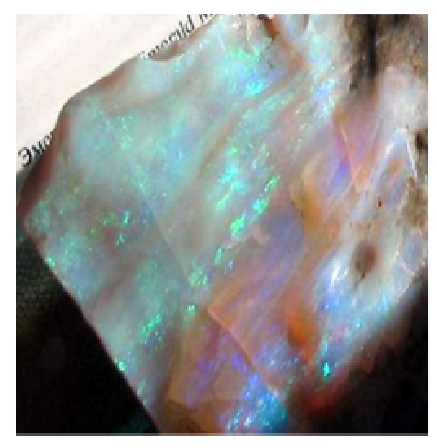

(a)

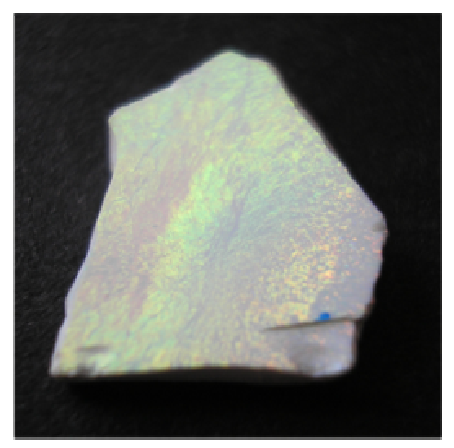

(b)

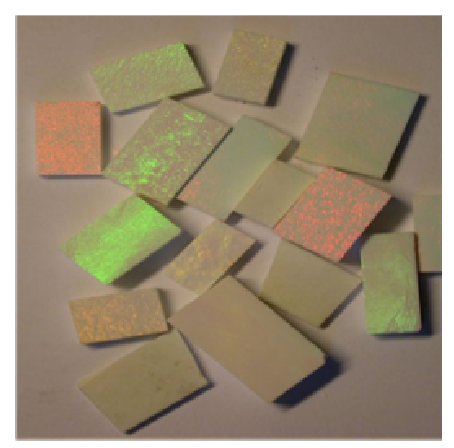

(c)

Fig. 1. Photographs of opal crystals: (a) natural opal (sample from the A E Fersman Mineralogical Museum of Russian Academy of Sciences; photo by A A Evseyev (http://geo.web.ru/druza/33_fo_6.htm)), (b) and (c) artificial opals provided by S M Samoilovich and used in our experiments.

The central position $(\lambda=580 \mathrm{~nm})$ and the spectral width $(\Delta \lambda=36 \mathrm{~nm})$ of the photonic stop band, the distance between (111) planes $(d=213 \mathrm{~nm})$ and the diameter of $\mathrm{SiO}_{2}$ globules $(a=261 \mathrm{~nm})$ for the volume SOPC samples were determined from the Bragg reflection spectra as described earlier [10]. For the film opals these parameters were as follows: $\lambda=600 \mathrm{~nm}, \Delta \lambda=66 \mathrm{~nm}, d=230 \mathrm{~nm}$ and $a=283 \mathrm{~nm}$. The fluorescence of the SOPC with R6G was excited by the radiation of LED Edixeon EDST-3LAx (the excitation light wavelength of $\lambda_{e x}=515 \mathrm{~nm}$ and the average power of $30 \mathrm{~mW}$ ). The samples with BBHQ were excited by the second harmonic $\left(\lambda_{e x}=532 \mathrm{~nm}\right)$ of the radiation from Q-switched YAG: $\mathrm{Nd}^{3+}$ laser (the pulse repetition rate of $8 \mathrm{kHz}$ and the average power from $50 \mathrm{~mW}$ to $200 \mathrm{~mW}$ ). The spectra were obtained with the use of modernized spectrometer DFS-12. They were detected within the aperture of $\leq 0.17 \pi$ sr in the direction $<111>$ of crystal. The corresponding useful signal was detected in the regime of photon counting with accumulation. 


\section{Results and Interpretation}

The fluorescence spectra of the R6G and BBHQ molecules are shown in Fig. 2. As seen from Fig. 2a, a suppression of the fluorescence intensity inside the region of 565-600 nm and a "blue" shift of short-wavelength tail of the spectrum by $10 \mathrm{~nm}$ take place for the R6G molecules embedded in the SOPC (see the blue curve). At the same time, the longwavelength tail of the fluorescence spectrum is not essentially altered. It should be pointed out that the exciting radiation is rather distant from the stop band position (the red area), while the R6G concentration is too small for changing essentially the effective refraction index of the SOPC and, consequently, for shifting the stop band. For the samples additionally infiltrated with colloidal silver (the green curve), one can observe a small shift of the fluorescence maximum towards longer wavelengths (by $\approx 3 \mathrm{~nm}$ ) as a slight reduction of the integral intensity, when compare with the spectrum for R6G molecules in the bare SOPC (the blue curve).

These effects might be linked with influence of the photonic stop band on the spectrum of secondary emission. First of all, this is revealed in partial suppression of the emission that is ballistically transported by photons through the opal structure and is caught in the stop-band region. The absence of total suppression (i.e., the presence of residual emission inside the stop-band region) is associated with influence of structural disordering on the photon DOS and with the corresponding increase in the transmission in this spectral region $[6,11]$. On the other hand, the short-wavelength emission of the R6G is reflected by the system of (111) planes at larger incidence angles, because the spectral position and the width of the stop band are also dependent on the angle between the light

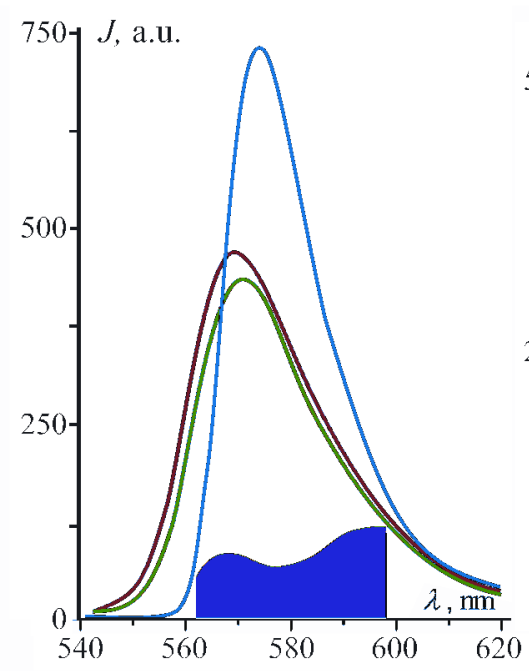

(a)

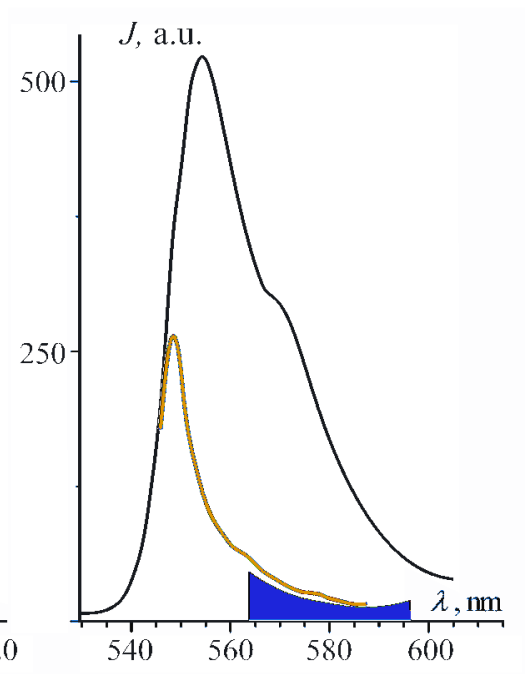

(b)

Fig. 2. Fluorescence spectra for the R6G (a) and $B B H Q$ (b) molecules: orange curve - R6G in methanol solution, blue - in bare SOPC, green - in Ag-infiltrated SOPC, black - BBHQ in optical cell (under the excitation power of $200 \mathrm{~mW}$ ) and cyan - embedded in film opal (under the excitation power of $50 \mathrm{~mW}$ ). Red areas denote the corresponding stop bands. 
propagation direction and the principal axes of crystal. Further, the emission directed along <111> appears as a result of multiple elastic scattering at the opal structure (the diffuse photon transport). This is the reason of "blue" shift of the short-wavelength tail in the fluorescence spectrum [7]. Infiltration of samples with colloidal silver leads to forming reflecting metallic layers among the Bragg planes (111), which cause an additional wide-range diffuse reflection and, consequently, decrease in the fluorescence intensity inside the "blue"-shift region.

The intensity distribution in the fluorescence spectra of BBHQ (the black curve in Fig. 2b) is similar to the emission spectrum with no proton transfer [12]. Similar to the emission of R6G molecules embedded in the SOPC, appreciable suppression of the spontaneous emission in the stop-band region is also observed for the embedded BBHQ molecules. Moreover, taking into account the relative difference in the powers typical for excitation of BBHQ in the optical cell and in the SOPC, one can point to amplification of the integral fluorescence intensity (see the cyan curve in Fig. 2b) occurring in the range of $535-545 \mathrm{~nm}$ for the BBHQ molecules embedded in the layered opals. This effect is caused by coincidence of the laser radiation wavelength with the stop-band edge and by accumulation of exciting photons in the sample due to their decreasing velocity. In this case, the efficiency of excitation of the BBHQ molecules in the photon crystal increases essentially because of a diffuse character of radiation propagation and increased probability of their interaction with the radiation field [6].

\section{Conclusion}

The effect of suppression of the spontaneous emission inside the stop-band region is experimentally proven for the molecules embedded in the volume of photonic crystal. The defects of structure of the synthetic opal photonic crystals are crucial for redistribution of the photon DOS in the region of photonic stop band and for understanding of the limited suppression. A "blue" shift of the fluorescence spectrum of R6G molecules embedded in the photonic crystal and its dependence on the infiltration with colloidal silver are detected. Amplification of the fluorescence spectra of BBHQ and their modification by the photon DOS are observed. It is shown that suppression of the light emission is not the only action of the photonic crystals. They can also enhance this emission.

\section{Acknowledgements}

The authors are much indebted to S M Samoilovich for generously providing the samples of synthetic opal photonic crystal and to professor V S Gorelik for stimulating and useful discussions.

\section{References}

1. Bykov V P, 1972. Spontaneous emission in a periodic structure. Sov. Phys. JETP 35: 269-273.

Ukr. J. Phys. Opt. 2008, V9, №3 
2. Yablonovitch E, 1987. Inhibited spontaneous emission in solid-state physics and electronics. Phys. Rev. Lett. 58: 2059-2062.

3. John S, 1987. Strong localization of photons in certain disordered dielectric superlattices. Phys. Rev. Lett. 58: 2486-2489.

4. Bogomolov V N, Gaponenko S V, Germanenko I N, Kapitonov A M, Petrov E P, Gaponenko N V, Prokofiev A V, Ponyavina A N, Silvanovich N I and Samoilovich S M, 1997. Photonic band gap phenomenon and optical properties of artificial opals. Phys Rev. E. 55: 7619-7625.

5. Gaponenko S V, Bogomolov V N, Petrov E P, Kapitonov A M, Eychmuller A A, Rogach I I, Gindele F and Woggon U, 2000. Spontaneous emission of organic molecules and semiconductor nanocrystals in a photonic crystal. J. Lumin. 87-89: 152-156.

6. Romanov S G and Fokin A V, 2003. Change of spontaneous emission in stop band of opal with magnified contrast of refraction index. Fiz. Tverd. Tela. 45: 419-423.

7. Bechger L, Lodahl P and Vos W L, 2005. Directional fluorescence spectra of laser dye in opal and inverse opal photonic crystals. J. Phys. Chem. B. 109: 9980-9988.

8. Emelchenko G A, Gruzincev A N, Kovalchuk M N, Maslov V M, Samarov E N, Yakimov E E, Barthou $\mathrm{C}$ and Zverkova I I, 2005. Opal-ZnO nanocomposites: structure and emission properties. Fiz. Tekhn. Poluprov. 39: 1375-1379.

9. Gorelik V S, 2007. Optics of globular photonic crystals. Kvant. Elektron. 37: 409432.

10. Podolskyy D S, Moiseyenko V N, Gorelik V S and Shvachich V G, 2006. Reflection spectra of bare and infiltrated with $\mathrm{Ba}\left(\mathrm{NO}_{3}\right)_{2}$ synthetic opal photonic crystals. Ukr. J. Phys. Opt. 7: 58-62.

11. Kaliteevskii M A, Nikolayev V V and Abram R A, 2005. Statistics of eigenstates and optical properties of one-dimensional disordered photonic crystals. Fiz. Tverd. Tela. 47: $1871-1880$.

12. Chayka K, Moiseyenko V and Mordzinski A, 2005. Luminescence of 2,5-bis(2benzoxazolyl)hydroquinone molecules adsorbed on copper island film. Ukr. J. Phys. Opt. 6: $128-132$. 